\title{
Erratum
}

Please be informed that on the following article:

\section{Complication and Management of Intestinal Stomas: In Indian Perspective}

Arghya Basu, Amit Kumar Gupta, Antora Choudhury, Soumika Biswas, Sukumar Maiti

published in the previous bulletin of Hellenic Journal of Surgery [2014, 86:3, pages 142-149], there is an inconsistency between the references and the numbering stated in the whole article, specifically the numbering stated in the article should change to fully comply with the references mentioned in the same chapter; i.e. number "4" should be number "1"; number "5" should be number "2" etc. 